\title{
Dynamic renormalization group study of a generalized continuum model of crystalline surfaces
}

\author{
Rodolfo Cuerno, ${ }^{1,}$ and Esteban Moro ${ }^{2}$, 间 \\ ${ }^{1}$ Departamento de Matemáticas $\&$ GISC, Universidad Carlos III de Madrid, \\ Avenida Universidad 30, E-28911 Leganés, Spain \\ ${ }^{2}$ Theoretical Physics Department, University of Oxford, \\ 1 Keble Road, OX1 3NP, United Kingdom
}

(Dated: February 1, 2008)

\begin{abstract}
We apply the Nozières-Gallet dynamic renormalization group (RG) scheme to a continuum equilibrium model of a $d$-dimensional surface relaxing by linear surface tension and linear surface diffusion, and which is subject to a lattice potential favoring discrete values of the height variable. The model thus interpolates between the overdamped sine-Gordon model and a related continuum model of crystalline tensionless surfaces. The RG flow predicts the existence of an equilibrium roughening transition only for $d=2$ dimensional surfaces, between a flat low-temperature phase and a rough high-temperature phase in the Edwards-Wilkinson (EW) universality class. The surface is always in the flat phase for any other substrate dimensions $d>2$. For any value of $d$, the linear surface diffusion mechanism is an irrelevant perturbation of the linear surface tension mechanism, but may induce long crossovers within which the scaling properties of the linear molecular-beam epitaxy equation are observed, thus increasing the value of the sine-Gordon roughening temperature. This phenomenon originates in the non-linear lattice potential, and is seen to occur even in the absence of a bare surface tension term. An important consequence of this is that a crystalline tensionless surface is asymptotically described at high temperatures by the EW universality class.
\end{abstract}

PACS numbers: 68.35.Rh, 64.60.Ak, 64.60.Ht, 81.10.Aj

*Electronic address: cuerno@math.uc3m.es

$\dagger$ Electronic address: moro@thphys.ox.ac.uk 


\section{INTRODUCTION}

The dynamics of growing surfaces [1], 2] has attracted great interest during the last decade. This is due both to the practical implications for the control of film quality in thin film production techniques, and to the fundamental questions it raises in areas of Physics such as spatially extended systems in the presence of fluctuations [3], or scale invariance in nonequilibrium systems [4]. It has been observed that a surface growing in the presence of fluctuations quite generically exhibits time and spatial scale invariance properties. Specifically, if $h(\mathbf{r}, t)$ denotes the surface height at time $t$ above a $d$-dimensional substrate position $\mathbf{r} \in \mathbb{R}^{d}$, the height-difference correlation function $G(r, t)=\left\langle\left(h\left(\mathbf{r}_{0}+\mathbf{r}, t\right)-h\left(\mathbf{r}_{0}, t\right)\right)^{2}\right\rangle$ grows as $G(r, t) \sim t^{2 \alpha / z}$ for $t^{1 / z} \ll r$ and scales as $G(r, t) \sim r^{2 \alpha}$ for $t^{1 / z} \gg r$, where $\alpha$ and $z$ are referred to as the roughness and dynamic exponents, respectively. These scale invariance properties imply that such rough surfaces share many properties with dynamic critical phenomena, which allows one to obtain useful information on the former from studies on the latter, and vice versa. A particularly interesting example is provided by the overdamped sine Gordon ( $\mathrm{sG}$ ) model, which describes the equilibrium fluctuations of a crystal surface and the features of its roughening transition [5]:

$$
\mu^{-1} \frac{\partial h}{\partial t}=F+\nu \Delta h-\frac{2 \pi V}{a_{\perp}} \sin \left(\frac{2 \pi h}{a_{\perp}}\right)+\sqrt{2 T \mu^{-1}} \eta(\mathbf{r}, t) .
$$

In Eq. (四), $\Delta$ is the substrate Laplacian, $\mu$ is the surface mobility, $F, \nu, V$ and $a_{\perp}$ are positive constants, $T$ is temperature, and $\eta$ is a zero mean, Gaussian white noise with correlations $\left\langle\eta(\mathbf{r}, t) \eta\left(\mathbf{r}^{\prime}, t^{\prime}\right)\right\rangle=\delta\left(\mathbf{r}-\mathbf{r}^{\prime}\right) \delta\left(t-t^{\prime}\right)$. The surface morphology of a crystal surface described by the $\mathrm{sG}$ model thus results from an interplay between linear surface tension, described by the term with coefficient $\nu$ in (1), a periodic potential favoring discrete interface values $h=n a_{\perp}$ with $n \in \mathbb{Z}$, and thermal fluctuations. Moreover, using the same arguments as in [6], for a non-zero value of the homogeneous driving (flux of aggregating particles) $F$ in (11), an alternative interpretation of the $\mathrm{sG}$ surface is as one minimizing surface area (for small values of the surface slope), subject to a flux of aggregating particles such that growth events of the height take place in integer values. In equilibrium $(F=0)$, the sG model is well known [7, 8, 9, 10] to feature a roughening transition when $d=2$ : for temperatures above a critical value $T \geq T_{R}^{\mathrm{sG}}$, the lattice potential is irrelevant and the surface is rough, in the sense quoted above for the height-difference correlation function. The corresponding exponent values are those of the Edwards-Wilkinson (EW) equation [11], which is simply the $V=0$ limit of Eq. (11), namely [12]

$$
\alpha_{\mathrm{EW}}=\frac{2-d}{2}, \quad z_{\mathrm{EW}}=2 .
$$

In the case $d=2$ considered, (2) amounts to $\alpha_{\mathrm{EW}}=0$, implying $G(r, t) \sim \log r$ for $r \ll$ $t^{1 / z}$. For temperatures smaller than $T_{R}^{\mathrm{sG}}$, the lattice potential dominates the large scale properties of the surface setting a finite correlation length $\xi$, beyond which the heightdifference correlation function $G(r>\xi, t)$ attains a constant value and hence the surface is flat. The $\mathrm{sG}$ roughening transition is of the Kosterlitz-Thouless class, and hence the continuum sG model is related to important discrete models such as the discrete Gaussian and the $\mathcal{F}$ models $[5,13]$.

As mentioned above, a practical domain which presents many instances of growing surfaces is the area of thin film production by techniques such as e.g. Molecular-Beam Epitaxy 
(MBE). In many MBE conditions [14], the main relaxation mechanism on the surface is surface diffusion, rather than surface tension. In this case, and again considering the additional effect of a periodic lattice potential, one is led naturally to the following model of a growing surface [15], henceforth referred to as the xMBE model

$$
\mu^{-1} \frac{\partial h}{\partial t}=F-\kappa \Delta^{2} h-\frac{2 \pi V}{a_{\perp}} \sin \left(\frac{2 \pi h}{a_{\perp}}\right)+\sqrt{2 T \mu^{-1}} \eta(\mathbf{r}, t) .
$$

In Eq. (3), $\kappa$ is a positive constant, in which case the corresponding term in the continuum equation does provide - again for small surface slopes - a surface diffusion relaxation mechanism [16]. All the other terms in Eq. (3) have the same meaning as in (11). Specifically, $F$ is related to the flow of adatoms onto the substrate. Thus, for $F \neq 0$, Eq. (3i) provides a non-equilibrium description of a growing surface which roughens under the effect of thermal fluctuations. In particular, as shown in [15], the surface described by Eq. (3) initially displays RHEED (Reflection High Energy Electron Diffraction) oscillations, akin to those observed experimentally [17]. For $F=0$, (3) describes the equilibrium fluctuations at temperature $T$ (we will consider a unit Boltzmann's constant) of a surface minimizing the energy functional (Hamiltonian)

$$
E(\kappa, V)=\int \mathrm{d}^{d} \mathbf{r}\left\{\frac{\kappa}{2}(\Delta h)^{2}+V\left[1-\cos \left(\frac{2 \pi h}{a_{\perp}}\right)\right]\right\} .
$$

Therefore, such a surface can be also thought of as minimizing surface curvature to linear approximation, thus having in principle zero surface tension, and to favor values of the height which are integer multiples of $a_{\perp}$. Note in this respect that (3) does not have the form of a continuity equation for the surface height, due to the form of the nonlinear and noise terms [18]. Hence, from the point of view of applications to MBE growth, the continuum xMBE model (3i) might be relevant for those situations in which nonconserved noise is expected to play a rôle, e.g. for length-scales larger than the typical diffusion length [19]. On the other hand, in a similar way as the $\mathrm{sG}$ equation can be seen as a continuum description of the discrete Gaussian model, Eq. (4) is a natural candidate for the continuum description of the discrete Laplacian roughening (Lr) model [20] on the square lattice [21]. The Lr model has been used to describe two-dimensional defect melting and as a model of tensionless surfaces such as membranes [22, 23]. Actually, Eq. (田) can indeed be obtained [24] as a continuum limit of the Lr model, albeit in a non-unique fashion.

Numerical simulations of Eq. (3) for $d=2$ [15, 25] show an equilibrium roughening transition, similar to that in the sG model. In the case of Eq. (3), the exponents characterizing the rough high temperature phase are numerically consistent with those obtained for the $V=0$ limit of Eq. (3) - the so called linear MBE (lMBE) equation 26, 27-, namely

$$
\alpha_{l \mathrm{MBE}}=\frac{4-d}{2}, \quad z_{l \mathrm{MBE}}=4,
$$

which in $d=2$ implies $\alpha_{l \mathrm{MBE}}=1$. However, given that in the sG system the periodic potential is known to contribute a correction to the surface tension term upon renormalization, the same phenomenon is expected to occur in the xMBE model, in which the bare surface tension is zero. Since surface diffusion is irrelevant in the presence of surface tension [14, 28], this argument together with the available numerical evidence [15, 25] led to the prediction that the scaling properties of the xMBE model in the high temperature phase are the same 
as those of the $\mathrm{sG}$ model. To date, and although more recent numerical data [29] confirm the existence of crossover behavior in the critical properties of model (田), direct numerical verification of EW scaling properties seems hard to achieve. Moreover, previous analytical studies [24], which employed a variational mean-field analysis successfully applied in [30] for the analysis of the sG roughening transition, have failed to reproduce EW scaling at high temperatures for the xMBE model. Specifically, the results obtained in [24] include an upper critical dimension $d_{c}=4$, below which model (3)-(国) displays a phase transition between a low temperature flat phase and a high temperature rough phase whose scaling behavior is that of the $l \mathrm{MBE}$ equation. However, the transition taking place is of first order for all substrate dimensions, $d \leq d_{c}$, while the numerical simulations of [15, 25] strongly suggest that, at least for $d=2$, the transition is of a continuous type. Besides, the generation of a surface tension term by the lattice potential referred to above is due to non-trivial coupling between different surface Fourier modes, which is unsufficiently accounted for by the variational mean-field description employed in [24]. Therefore it is natural to try improve on the mean-field approximation of Ref. [24] and explore the scale invariant properties of the system near the phase transition point through the use of the renormalization group (RG).

In this paper we consider the following generalized continuum model of surfaces subject to a periodic lattice potential:

$$
\begin{aligned}
\mu^{-1} \frac{\partial h}{\partial t}= & F+\nu \Delta h-\kappa \Delta^{2} h-\frac{2 \pi V}{a_{\perp}} \sin \left(\frac{2 \pi h}{a_{\perp}}\right) \\
& +\sqrt{2 T \mu^{-1}} \eta(\mathbf{r}, t) .
\end{aligned}
$$

This equation obviously features both Eqs. (ID) [sG model] and (3) [xMBE model] as special cases. Moreover, the linear limit $V=0$ [14, 28] of this equation has been observed to accurately describe growth experiments of copper aggregates by electrochemical deposition in the presence of organic additives [31]. For the sake of simplicity, in this work we will consider this generalized model in the absence of driving $(F=0)$, in which case Eq. (6) describes the equilibrium fluctuations of a surface with Hamiltonian

$$
E_{g}(\nu, \kappa, V)=\int \mathrm{d}^{d} \mathbf{r}\left\{\frac{\nu}{2}(\nabla h)^{2}+\frac{\kappa}{2}(\Delta h)^{2}+V\left[1-\cos \left(\frac{2 \pi h}{a_{\perp}}\right)\right]\right\} .
$$

In order to study the critical properties of the xMBE model in $d$ substrate dimensions, we will extend the dynamic RG approach devised by Nozières and Gallet (NG) for the $d=2 \mathrm{sG}$ model [8] (see also a detailed account in [9]) to the generalized system (6). There are two reasons for our pursuing this approach: (i) as anticipated above, in the RG analysis of (3)-(位) a finite (non-zero) surface tension term needs to be allowed for, given that it is generated in any perturbative scheme even if its bare amplitude is zero; (ii) a static renormalization group study of the equilibrium system (仼) is ill-defined in some parameter ranges due to divergent integrals [25], similarly to the $\mathrm{sG}$ case [8]. Still, the static RG study will provide us, via the appropriate generalization, with the correct expansion of the model non-linearity in terms of relevant operators through the use of Kadanoff's operator product expansion (OPE) [32, as was accomplished in [8, 33] for the sG model. In any case, the results to be obtained from the dynamic RG study that follows will also cover the case of a system minimizing both surface area and surface curvature, and will in particular allow us to analyze how the standard sG roughening transition is modified when an additional surface diffusion term 
is considered. We will finally consider the renormalization properties of the xMBE model, which corresponds to a specific choice of bare parameters within this generalized framework, and will compare the conclusions obtained with those from both the variational approach [24] and numerical simulations [15, 25]. To our knowledge, ours is the first RG approach to the xMBE model as formulated by (3)-(四), and it may contribute to the elucidation of the existence and nature of the phase transition in this and related systems, as the Lr model. There also exist static and dynamic RG studies of similar systems. Specifically, the equilibrium properties of a model which is different from (33)-(偖) but is believed to provide the continuum description of the Lr model on the triangular lattice, have been analyzed in [34], and its dynamical properties have been obtained in [35] and references therein. Within the rough MBE surfaces context, the dynamic properties of the conserved sG model have been studied both under conserved [18, 36] and non-conserved [18, 37] noise. Finally, a Langevin equation believed to describe a restricted curvature model [38] has been analyzed in [39 by using RG techniques.

This paper is organized as follows. In Sec. II we apply the dynamic RG scheme of NG to Eq. (6). The parameter flow thus obtained for this generalized model is studied in Sec. [IIA as a function of the substrate dimension $d$. The special limit of Eq. (6) corresponding to crystalline tensionless surfaces, Eq. (3), requires additional considerations of a technical nature, and is deferred to Sec. IIIB. Finally, Sec. IV is devoted to further discussion of the results obtained in the previous sections, and to summarizing our conclusions. We additionally provide two appendices. In Appendix $\mathrm{A}$ we detail, following [8, 33], the OPE which is needed in the dynamic RG in order to perform the appropriate expansion of the lattice potential in (6) into relevant operators. Appendix B closes with a discussion of the specific way in which the roughening transition of the sG model generalizes into that to be obtained in Sec. IIIA for (6)-(7).

\section{DYNAMIC RENORMALIZATION GROUP ANALYSIS}

This section is devoted to the analysis of Eq. (6) in the equilibrium case $F=0$, employing the dynamic RG scheme of $\mathrm{NG}[\mathbf{8}, 9$. In this scheme, a coarse graining procedure is performed over the microscopic modes of the noise term. Namely, the noise is split into two statistically independent parts, $\eta=\bar{\eta}+\delta \eta$, such that the total noise power spectrum is the sum of the corresponding contributions. Here $\delta \eta(\mathbf{r}) \equiv \int_{\bar{\Lambda}}^{\Lambda} \mathrm{d}^{2} \mathbf{k} \mathrm{e}^{\mathrm{ikr}} \hat{\eta}(\mathbf{k})$, where $\Lambda$ is a momentum cut-off related e.g. to atomic positions on the substrate, $\bar{\Lambda}=\mathrm{e}^{-\varepsilon} \Lambda$ with $\varepsilon$ a small parameter, and $\hat{\eta}(\mathbf{k})$ is the spatial Fourier transform of the noise. Then, microscopic fluctuations are integrated out by defining $\bar{h} \equiv\langle h(\bar{\eta}+\delta \eta)\rangle_{\delta \eta}$ and $\delta h \equiv h-\bar{h}$, and by seeking an equation of motion for the thus defined long distance modes $\bar{h}$. The result will be an equation with the same shape as (6), but with new (renormalized) coefficients, which are sensitive to the microscopic fluctuations $\delta \eta$ by the action of the nonlinearities. Specifically, the dynamic equations for the $\bar{h}$ and $\delta h$ modes read

$$
\begin{aligned}
\mu^{-1} \frac{\partial \bar{h}}{\partial t}= & \nu \Delta \bar{h}-\kappa \Delta^{2} \bar{h}-\langle\Phi(\bar{h}, \delta h)\rangle_{\delta \eta} \\
& +\sqrt{2 D} \bar{\eta}(\mathbf{r}, t), \\
\mu^{-1} \frac{\partial \delta h}{\partial t}= & \nu \Delta \delta h-\kappa \Delta^{2} \delta h-\left[\Phi(\bar{h}, \delta h)-\langle\Phi(\bar{h}, \delta h)\rangle_{\delta \eta}\right] \\
& +\sqrt{2 D} \delta \eta(\mathbf{r}, t),
\end{aligned}
$$


where we have defined $D \equiv T \mu^{-1}$, and we have introduced $\Phi(\bar{h}, \delta h) \equiv\left(2 \pi V / a_{\perp}\right) \sin [2 \pi(\bar{h}+$ $\left.\delta h) / a_{\perp}\right]$. In order for (8) to be a closed equation in $\bar{h}$, we need to solve for $\delta h$ in (9) and introduce the result into (8). The formal solution of (9) reads

$$
\begin{aligned}
\delta h(\mathbf{r}, t)= & \int \mathrm{d}^{d} \mathbf{r}^{\prime} \int_{-\infty}^{t} \mathrm{~d} t^{\prime} \chi_{0}\left(\mathbf{r}-\mathbf{r}^{\prime}, t-t^{\prime}\right) \\
& \times\left[\sqrt{2 D} \delta \eta\left(\mathbf{r}^{\prime}, t^{\prime}\right)+\Phi^{\prime}-\left\langle\Phi^{\prime}\right\rangle_{\delta \eta}\right],
\end{aligned}
$$

where the primed notation denotes dependence on the $\mathbf{r}^{\prime}, t^{\prime}$ variables, and the $d$-dimensional free propagator reads

$$
\chi_{0}(\mathbf{r}, t)=\int \frac{\mathrm{d}^{d} k \mathrm{~d} \omega}{(2 \pi)^{d+1}} \frac{\mathrm{e}^{\mathrm{i}(\mathbf{k r}-\omega t)}}{\nu k^{2}+\kappa k^{4}-\mathrm{i} \omega \mu^{-1}} .
$$

Due to the non-linear lattice potential, an explicit solution of (9) can only be obtained by performing a perturbative expansion in powers of $V$. Thus, defining

$$
\delta h(\mathbf{r}, t)=\delta h^{(0)}(\mathbf{r}, t)+V \delta h^{(1)}(\mathbf{r}, t)+\mathcal{O}\left(V^{2}\right),
$$

we obtain

$$
\begin{aligned}
\delta h^{(0)}(\mathbf{r}, t)= & \int \mathrm{d}^{d} \mathbf{r}^{\prime} \int_{-\infty}^{t} \mathrm{~d} t^{\prime} \chi_{0}\left(\mathbf{r}-\mathbf{r}^{\prime}, t-t^{\prime}\right) \\
& \times \sqrt{2 D} \delta \eta\left(\mathbf{r}^{\prime}, t^{\prime}\right), \\
\delta h^{(1)}(\mathbf{r}, t)= & -\frac{4 \pi^{2}}{a_{\perp}^{2}} \int \mathrm{d}^{d} \mathbf{r}^{\prime} \int_{-\infty}^{t} \mathrm{~d} t^{\prime} \chi_{0}\left(\mathbf{r}-\mathbf{r}^{\prime}, t-t^{\prime}\right) \\
& \times \delta h^{\prime(0)} \cos \left(\frac{2 \pi \bar{h}^{\prime}}{a_{\perp}}\right) .
\end{aligned}
$$

Using $\sin (a+b)=\sin a \cos b+\cos a \sin b$, and within our perturbation expansion, we can now evaluate in (8)

$$
\begin{aligned}
\langle\Phi(\bar{h}, \delta h)\rangle_{\delta \eta}= & \frac{2 \pi V}{a_{\perp}}\left[1-\frac{2 \pi^{2}}{a_{\perp}^{2}}\left\langle\left(\delta h^{(0)}\right)^{2}\right\rangle_{\delta \eta}\right. \\
& \left.-\frac{4 \pi^{2} V}{a_{\perp}^{2}}\left\langle\delta h^{(0)} \delta h^{(1)}\right\rangle_{\delta \eta}+\mathcal{O}\left(V^{2}\right)\right] \\
& \times \sin \left(\frac{2 \pi \bar{h}}{a_{\perp}}\right)
\end{aligned}
$$

which has the form

$$
\langle\Phi\rangle_{\delta \eta}(\bar{h})=V \Phi^{(1)}(\bar{h})+V^{2} \Phi^{(2)}(\bar{h})+\mathcal{O}\left(V^{3}\right)
$$

with

$$
\begin{aligned}
\Phi^{(1)}(\bar{h})= & \frac{2 \pi}{a_{\perp}}\left[1-\frac{(2 \pi)^{2-d} \mathcal{S}_{d} T \Lambda^{d-2} \varepsilon}{2 a_{\perp}^{2}\left(\nu+\kappa \Lambda^{2}\right)}\right] \\
& \times \sin \left(\frac{2 \pi \bar{h}}{a_{\perp}}\right), \\
\Phi^{(2)}(\bar{h})= & -\frac{8 \pi^{3}}{a_{\perp}^{3}}\left\langle\delta h^{(0)} \delta h^{(1)}\right\rangle_{\delta \eta} \sin \left(\frac{2 \pi \bar{h}}{a_{\perp}}\right),
\end{aligned}
$$


where $\mathcal{S}_{d}=2 \pi^{d / 2} / \Gamma(d / 2)$ is the surface area of the unit hypersphere in $d$ dimensions, $\Gamma(\cdot)$ being Euler's Gamma function. The shape of (17) already reflects the fact that we have considered an infinitesimal shell of microscopic noise modes of width $\varepsilon$. We can write (18) more explicitly as

$$
\begin{aligned}
\Phi^{(2)}(\bar{h})= & \frac{32 \pi^{5}}{a_{\perp}^{5}} \int \mathrm{d}^{d} \mathbf{r}^{\prime} \int_{-\infty}^{t} \chi_{0}\left(\mathbf{r}-\mathbf{r}^{\prime}, t-t^{\prime}\right) \\
& \times\left\langle\delta h^{(0)} \delta{h^{\prime}}^{(0)}\right\rangle_{\delta \eta} \sin \left(\frac{2 \pi \bar{h}}{a_{\perp}}\right) \cos \left(\frac{2 \pi \bar{h}^{\prime}}{a_{\perp}}\right) .
\end{aligned}
$$

Neglecting higher order harmonics of the lattice potential, $\sin \left(2 \pi \bar{h} / a_{\perp}\right) \cos \left(2 \pi \bar{h}^{\prime} / a_{\perp}\right) \simeq$ $\frac{1}{2} \sin \left[2 \pi\left(\bar{h}-\bar{h}^{\prime}\right) / a_{\perp}\right]$. Further, we can use the results for the OPE of the lattice potential obtained in Appendix A, and a Taylor expansion to obtain

$$
\begin{aligned}
\Phi^{(2)}(\bar{h}) \simeq & \frac{32 \pi^{6}}{a_{\perp}^{6}} \mathcal{S}_{d} \int_{0}^{\infty} \rho^{d-1} \mathrm{~d} \rho \int_{0}^{\infty} \mathrm{d} \tau \chi_{0}(\rho, \tau) \\
& \times\left\{\tau \frac{\partial \bar{h}}{\partial t}-\frac{\rho^{2}}{2 d} \Delta \bar{h}-\frac{\rho^{4}}{8 d(d+2)} \Delta^{2} \bar{h}\right\} \\
& \times\left\langle\delta h^{(0)} \delta h^{\prime(0)}\right\rangle_{\delta \eta} \exp \left[-\frac{2 \pi T}{a_{\perp}} \phi(\rho, \tau, \nu, \kappa, \mu)\right],
\end{aligned}
$$

where we have defined $\boldsymbol{\rho} \equiv \mathbf{r}-\mathbf{r}^{\prime}$ and $\tau \equiv t-t^{\prime}$, the $d$-dimensional free propagator reads

$$
\chi_{0}(\rho, \tau)=\frac{\mu}{(2 \pi)^{d / 2}} \int_{0}^{\Lambda} \mathrm{d} k \frac{k^{d / 2}}{\rho^{d / 2-1}} J_{d / 2-1}(\rho k) \mathrm{e}^{-\left(\nu k^{2}+\kappa k^{4}\right) \mu \tau},
$$

and we have introduced

$$
\begin{aligned}
\phi(\rho, \tau, \nu, \kappa, \mu) & \equiv \frac{\mathcal{S}_{d}}{(2 \pi)^{d-1}} \int_{0}^{\Lambda} \mathrm{d} k \frac{k^{d-1}}{\nu k^{2}+\kappa k^{4}}[1- \\
& \left.-\frac{\Gamma(d / 2) J_{d / 2-1}(\rho k)}{(\rho k / 2)^{d / 2-1}} \mathrm{e}^{-\left(\nu k^{2}+\kappa k^{4}\right) \mu \tau}\right],
\end{aligned}
$$

with $J_{n}(x)$ being the $n$-th order Bessel function of the first kind. Finally, using the results of Eqs. (17) and (20), Eq. (16) has the form

$$
\begin{aligned}
\langle\Phi\rangle_{\delta h}(\bar{h})= & (V+\varepsilon \delta V) \sin \left(\frac{2 \pi \bar{h}}{a_{\perp}}\right)-\varepsilon \delta \mu^{-1} \frac{\partial \bar{h}}{\partial t} \\
& +\varepsilon \delta \nu \Delta \bar{h}+\varepsilon \delta \kappa \Delta^{2} \bar{h}+\mathcal{O}\left(V^{3}\right),
\end{aligned}
$$

where the corrections $\delta \nu, \delta \kappa$, etc. are implicitly defined. Inserting the result of Eq. (23) into Eq. (8), we obtain that, to $V^{2}$ order, the long distance modes $\bar{h}$ obey an equation with the same shape as Eq. (6), namely,

$$
\tilde{\mu}^{-1} \frac{\partial \bar{h}}{\partial t}=\tilde{\nu} \Delta \bar{h}-\tilde{\kappa} \Delta^{2} \bar{h}-\frac{2 \pi \tilde{V}}{a_{\perp}} \sin \left(\frac{2 \pi \bar{h}}{a_{\perp}}\right)+\sqrt{2 D} \bar{\eta}(\mathbf{r}, t),
$$


but with new coefficients $\tilde{\nu} \equiv \nu+\varepsilon \delta \nu, \tilde{\kappa} \equiv \kappa+\varepsilon \delta \kappa, \tilde{\mu}^{-1} \equiv \mu^{-1}+\varepsilon \delta \mu^{-1}$, and $\tilde{V} \equiv V+\varepsilon \delta V$. In order to recover the original Fourier mode cut-off $\Lambda$, we now rescale variables as

$$
\begin{aligned}
\mathbf{r} & \rightarrow \mathbf{r}^{\prime}=\mathbf{r} / b \\
\bar{h} & \rightarrow \bar{h}^{\prime}=\bar{h} b^{-\alpha} \\
t & \rightarrow t^{\prime}=b^{-z} t
\end{aligned}
$$

where $b=\mathrm{e}^{\varepsilon}$, and we thus get

$$
\bar{\mu}^{-1} \frac{\partial \bar{h}}{\partial t}=\bar{\nu} \Delta \bar{h}-\bar{\kappa} \Delta^{2} \bar{h}-\frac{2 \pi \bar{V}}{a_{\perp}} \sin \left(\frac{2 \pi \bar{h}}{a_{\perp}}\right)+\sqrt{2 \bar{D}} \bar{\eta}(\mathbf{r}, t),
$$

with coefficients $\bar{\nu}=\tilde{\nu} b^{z-2}, \bar{\kappa}=\tilde{\kappa} b^{z-4}, \bar{V}=\tilde{V} b^{z-2 \alpha}, \bar{\mu}^{-1}=\tilde{\mu}^{-1}$, and $\bar{D}=D b^{z-2 \alpha-d}$. Finally, for an infinitesimal $\varepsilon=\mathrm{d} l$ and expanding $\bar{\nu}(\varepsilon)$, etc., to first order in $\varepsilon$, we obtain the dynamic RG flow to $V^{2}$ order:

$$
\begin{aligned}
\frac{\mathrm{d} \nu}{\mathrm{d} l}= & (z-2) \nu \\
& +\frac{(2 \pi)^{6-d} \mathcal{S}_{d} T \Lambda^{d-6}}{4 d a_{\perp}^{6}\left(\nu+\kappa \Lambda^{2}\right)^{2}} V^{2} B^{(2,0)}(\nu, \kappa), \\
\frac{\mathrm{d} \kappa}{\mathrm{d} l}= & (z-4) \kappa \\
& -\frac{(2 \pi)^{6-d} \mathcal{S}_{d} T \Lambda^{d-8}}{16 d(d+2) a_{\perp}^{6}\left(\nu+\kappa \Lambda^{2}\right)^{2}} V^{2} B^{(4,0)}(\nu, \kappa), \\
\frac{\mathrm{d} V}{\mathrm{~d} l}= & (z-2 \alpha) V-\frac{(2 \pi)^{2-d} \mathcal{S}_{d} T \Lambda^{d-2}}{2 a_{\perp}^{2}\left(\nu+\kappa \Lambda^{2}\right)} V, \\
\frac{\mathrm{d} \mu^{-1}}{\mathrm{~d} l}= & \frac{(2 \pi)^{6-d} \mathcal{S}_{d} T \Lambda^{d-6}}{2 a_{\perp}^{6}\left(\nu+\kappa \Lambda^{2}\right)^{3}} V^{2} \mu^{-1} B^{(0,1)}(\nu, \kappa), \\
\frac{\mathrm{d} D}{\mathrm{~d} l}= & (z-2 \alpha-d) D, \\
\frac{\mathrm{d} a_{\perp}}{\mathrm{d} l}= & -\alpha a_{\perp},
\end{aligned}
$$

with

$$
\begin{aligned}
B^{(n, m)}(\nu, \kappa) & \equiv \int_{0}^{\infty} \mathrm{d} \tilde{\rho} \tilde{\rho}^{n+1} \int_{0}^{\infty} \mathrm{d} \tau \mathrm{e}^{-\tau} \tau^{m} J_{d / 2-1}(\tilde{\rho}) G(\tilde{\rho}, \tau, \nu, \kappa) \exp \left[-\frac{2 \pi T}{a_{\perp}^{2}} \phi(\tilde{\rho}, \tau, \nu, \kappa, \mu 03] 5,\right) \\
G(\tilde{\rho}, \tau, \nu, \kappa) & \equiv \int_{0}^{1} \mathrm{~d} \tilde{k} \tilde{k}^{d / 2} J_{d / 2-1}(\tilde{\rho} \tilde{k}) \exp \left[-\tau \frac{\nu \tilde{k}^{2}+\kappa \Lambda^{2} \tilde{k}^{4}}{\nu+\kappa \Lambda^{2}}\right],
\end{aligned}
$$

where $\tilde{\rho} \equiv \rho \Lambda$. It is worth noting that the flow of the mobility $\mu$ is enslaved to that of all other system parameters, which allows us to neglect its evolution under (29)-(34) in the rest of the paper. Moreover, in order to preserve fluctuation-dissipation by this RG procedure [8, 9] we need to impose the exponent relation $z=2 \alpha+d$ in the flow (29)-(34). Note that this relation (termed hyperscaling [40] in the studies of kinetic roughening) holds exactly at two 
fixed lines of (29)-(34), which are the two linear limits of (6), namely the EW $[V=\kappa=0$, see Eq. (2) $]$ and $l \mathrm{MBE}[V=\nu=0$, see Eq. (5)] equations. Both systems can be interpreted as describing equilibrium fluctuations, governed by the corresponding Hamiltonians. We also remark that, as anticipated in Sec. I, it is clear from Eq. (29) that, if we consider the $\mathrm{RG}$ flow for the xMBE model (3)-(四) in which there is no bare surface tension, the lattice potential does generate it under RG iterarion.

Finally, we find it more convenient to express the flow in the dimensionless variables

$$
x \equiv \frac{2 a_{\perp}^{2}}{\pi T}\left(\nu+\kappa \Lambda^{2}\right), \quad y \equiv \frac{4 \pi V}{T \Lambda^{2}}, \quad K \equiv \frac{\kappa a_{\perp}^{2} \Lambda^{2}}{\pi T} .
$$

Thus, we have

$$
\begin{aligned}
\frac{\mathrm{d} x}{\mathrm{~d} l}= & (d-2) x-4 K \\
& +\frac{4}{d} \frac{y^{2}}{x^{2}}\left[\tilde{B}^{(2)}(x, K)-\frac{1}{4(d+2)} \tilde{B}^{(4)}(x, K)\right] \\
\frac{\mathrm{d} y}{\mathrm{~d} l}= & 2 y\left[\frac{d}{2}-\frac{\mathcal{S}_{d}}{(2 \pi)^{d-1}} \frac{\Lambda^{d-2}}{x}\right] \\
\frac{\mathrm{d} K}{\mathrm{~d} l}= & (d-4) K-\frac{1}{2 d(d+2)} \frac{y^{2}}{x^{2}} \tilde{B}^{(4)}(x, K)
\end{aligned}
$$

where

$$
\begin{aligned}
\tilde{B}^{(n)}(x, K) & \equiv \frac{\mathcal{S}_{d} \Lambda^{d-2}}{(2 \pi)^{d-1}} \int_{0}^{\infty} \mathrm{d} \tilde{\rho} \tilde{\rho}^{n+1} \int_{0}^{\infty} \mathrm{d} \tau \mathrm{e}^{-\tau} J_{d / 2-1}(\tilde{\rho}) \tilde{G}(\tilde{\rho}, \tau, x, K) \mathrm{e}^{-2 \tilde{\phi}(\tilde{\rho}, \tau, x, K)} \\
\tilde{G}(\tilde{\rho}, \tau, x, K) & \equiv \int_{0}^{1} \mathrm{~d} \tilde{k} \tilde{k}^{d / 2} J_{d / 2-1}(\tilde{k} \tilde{\rho}) \mathrm{e}^{-2 \tau\left[(x / 2-K) \tilde{k}^{2}+K \tilde{k}^{4}\right] / x} \\
\tilde{\phi}(\tilde{\rho}, \tau, x, K) & \equiv \frac{\mathcal{S}_{d} \Lambda^{d-2}}{(2 \pi)^{d-1}} \int_{0}^{1} \mathrm{~d} \tilde{k} \frac{\tilde{k}^{d-1}}{\left(x / 2-K \tilde{k}^{2}+K \tilde{k}^{4}\right.}\left[1-\frac{\Gamma(d / 2) J_{d / 2-1}(\tilde{k} \tilde{\rho})}{(\tilde{k} \tilde{\rho} / 2)^{d / 2-1}} \mathrm{e}^{-2 \tau\left[(x / 2-K) \tilde{k}^{2}+K \tilde{k}^{4}\right] /(x)}\right]
\end{aligned}
$$

Note that the convergence properties of the integral $\tilde{\phi}(\tilde{\rho}, \tau, x, K)$ defined above depend on the substrate dimensionality $d$ and on the parameter values. Specifically, for $d=2$ and the condition associated with the xMBE model (3), namely, $x=2 K$, the integral diverges logarithmically at the lower limit. This divergence originates in the fact that correlation functions do not have a well-defined thermodynamic limit for the $l \mathrm{MBE}$ model in $d=2$ [14.

The dynamic RG flow (38)-(40) just obtained generalizes that in [8, 9] for the case of a finite surface diffusion term, and for any value of the substrate dimension $d$. The sineGordon case is retrieved simply by setting $\kappa=0$ and neglecting the higher order contribution $\tilde{B}^{(4)}(x, 0)$, related with the RG flow of the surface diffusion term. Actually, this integral will allow us to study in the next section the effect of such relaxation mechanism on the sG roughening transition.

\section{DISCUSSION OF THE RG FLOW}

The fixed point structure of the RG flow (38)-(40) depends on substrate dimensionality $d$, and thus so do the critical properties of model (6)-(7). In what follows we will restrict ourselves to dimensions $d \geq 2$ in which phase transitions are expected to occur. 
For general values of $d$, there are no proper non-trivial fixed points of (38)-(40), but rather regions in the $(x, y, K)$ phase space which are invariant under the $\mathrm{RG}$ flow. These correspond to the two significant linear limits of the generalized model, namely the EW line, $\Gamma_{\mathrm{EW}}$, and the $l \mathrm{MBE}$ line, $\Gamma_{l \mathrm{MBE}}$, defined by

$$
\begin{aligned}
\Gamma_{\mathrm{EW}} & =\{(x, y, K): y=K=0, x \neq 0\}, \\
\Gamma_{l \mathrm{MBE}} & =\{(x, y, K): x=2 K, y=0\} .
\end{aligned}
$$

However, for the special dimensions $d=2$ and $d=4$, respectively, these regions become lines of fixed points.

\section{A. Generalized model}

We start by analizing generic features of the RG flow as a function of substrate dimension $d$, in the case in which the bare values of surface diffusion and surface tension are both nonzero. The special initial condition (for the RG flow) in which there is no bare surface tension (xMBE model) will be considered in detail in Sec. ПIIB.

\section{1. $d=2$ case}

Obviously, the case which is most interesting from the physical point of view is that of a two-dimensional substrate. As stated above, now the invariant EW region $\Gamma_{\mathrm{EW}}$ actually becomes the well-known sG line of fixed points [5, 9]. An important point along this line is $x=1$, where the flow of the lattice potential $y$ changes stability, see Appendix $\mathrm{B}$. If the bare value of the surface diffusion $\kappa(l=0)=0$, as in the sG model, numerical integration of (38)(40) shows that the flow essentially remains on the $(x, y)$ plane and thus completely reduces to that of the sG system, as seen in panel (a) on Fig. 1: for high $T$ the flow is towards the $y=0$ axis, the interface being rough and characterized by the scaling properties of the EW equation. For low $T$ values, the lattice potential $y$ grows indefinitely upon iteration of the RG flow, drawing the system into the $T=0$ limit for which the interface is flat. Thus, there exists a roughening transition between these flat and rough phases. Numerically, we estimate the critical temperature as the value characterizing the trajectory that separates between flow onto the $y=0$ line and flow towards increasing $y$. We thus obtain $T_{R}^{s G} \simeq 0.725 \pm 0.05$ for $V=1, a_{\perp}=1, \nu=1, \kappa=0$, which is close to the value for the flow equations of the pure sG model [5, 8], $T_{R}^{s G}=2 / \pi \simeq 0.64$. On the other hand, as we see in panels (b) and (c) on Fig. 1, in general a small initial surface diffusion shifts the roughening temperature to higher values. The reason for this effect is that, as studied in [14, 28] when $V=0$, even though surface diffusion is irrelevant relative to surface tension in the hydrodynamic limit, an initial value $\kappa(0) \neq 0$ introduces a crossover length scale, $L_{\times}(l)=[\kappa(l) / \nu(l)]^{1 / 2}$, below which surface diffusion is the relevant relaxation mechanism. Once the coarse-graining procedure has overcome this scale, $\kappa(l)$ renormalizes to zero (see Appendix B) and the flow takes place on the $\mathrm{sG}(x, y)$ plane with a $\nu$ coefficient which differs from its bare value. From there on the flow is effectively as that of the pure sG model. The results shown on panel (d) of Fig. 11 correspond to a numerical simulation of the sG model [Eq. (6) with $\kappa \equiv 0, F=0]$ for $\mu=\nu=V=a_{\perp}=1$, and of Eq. (6) for $\kappa=0.5$, for both of which we have computed the specific heat [defined as $\chi_{E} \equiv\left(\left\langle E_{g}^{2}\right\rangle-\left\langle E_{g}\right\rangle^{2}\right) /\left(T L^{2}\right)$, with $E_{g}$ as in (7)] for 
several substrate lateral sizes $L$ and periodic boundary conditions. Clearly, the roughening transition temperature - which is preceded by a peak in $\chi_{E}$ as in the pure sG model [42]shifts to higher values for $\kappa \neq 0$. Integrating numerically the RG flow (38)-(40), we obtain $T_{R}^{s G}(\kappa=0.5) \simeq 1.35 \pm 0.05$.

\section{2. $d>2$ cases}

In order to inquire about the upper critical dimension, $d_{c}$, of model (岢)-(而), we consider values of $d$ above the physical two-dimensional case. An important consequence of this is that the points on the EW line $\Gamma_{\mathrm{EW}}$ are not fixed any longer under the RG iteration. This already signals the final result that, in these dimensions, there is no proper phase transition. Rather, the only existing phase is the flat low temperature one. Before justifying this result in detail, let us note that the EW equation already predicts a flat surface for $d>2$ [1, 2, 12], and therefore when adding a lattice potential to the surface tension term in the sG equation, the result that $d_{c}=2$ is the corresponding upper critical dimension [32, 43] does not come as a surprise. In the generalized model (6)-(17), similar results to those in the previous section indicate that the same value $d_{c}=2$ actually occurs: for $2<d<4$, it is clear from Eq. (40) that $K(l)$ still decreases exponentially under the RG iterarion, and again the flow becomes essentially that of the $\mathrm{sG}$ model in the corresponding dimension, thus predicting a flat morphology. In Fig. 2(a) we show as an illustration results of a numerical integration of (38)-(40) for $d=3$. As is clear from the figure [for this, it is useful to note the projection of the RG flow lines onto the $(x, y)$ plane], the flow is eventually towards large $y$ values for all temperatures. Note also that the $K(l)$ value may in some cases become negative, signalling a non-physical instability. We attribute this effect [also apparent on panels (b) and (c) of Fig. 2] to limitations of our $\mathcal{O}\left(V^{2}\right)$ approximation to the $\mathrm{RG}$ flow.

The value $d=4$ is marginal for the surface diffusion term in Eq. (6). In an analogous way to the rôle of $d=2$ for the EW equation [see Eq. (2)], the $l \mathrm{MBE}$ equation predicts a roughness exponent $\alpha=0$ for $d=4$ [logarithmic behavior of $G(r, t)$, see Eq. (5)] and a flat morphology for $d>4$ [1, 2]. Even though the decay of $K(l)$ under the RG flow (38)-(40) might not be so fast as in smaller dimensions, it nevertheless occurs, see Fig. 2(b), with the result of an effective $\mathrm{sG}$ behavior in $d=4$ dimensions, again corresponding to a flat morphology. Finally, as illustrated in Fig. 2(c) for $d=5$, if $d>4$ all coefficients in the RG flow grow under iteration. In particular the lattice potential $y$ increases indefinitely and thus the surface morphology is dictated by the behavior of the $T \rightarrow 0$ limit, namely again there is no phase transition and the surface is flat.

\section{B. Crystalline tensionless surfaces}

As remarked above, within the RG flow of the generalized model (6)-(7), the case corresponding to the xMBE model (3)-(四) simply amounts to a specific condition on the bare parameters, namely they lie on the plane $x(0)=2 K(0), y(0) \neq 0$, thus implying $\nu(0)=0$. In this section we thus study the RG flow for the xMBE model separately for different values of the substrate dimension. 


\section{1. $d=2$ case}

In the physical $d=2$ case, and due to the divergencies in the correlation functions mentioned above, this very condition induces a trivial RG flow, since the $\tilde{B}^{(n)}$ integrals in (41) become identically zero. The linear combination $x-2 K-$ which is proportional to the surface tension-, is a constant under the RG flow. Equations (38)-(40) can be exactly solved to show that any initial condition on the $x=2 K$ plane tends to the origin for $l \rightarrow \infty$. For large enough but finite $l$, the surface scales as the $l \mathrm{MBE}$ equation independently of the value of $T$, and thus there is no temperature driven phase transition, which contradicts the results of e.g. numerical simulations of Eq. (3) for two-dimensional substrates [15]. Thus, some kind of integral regularization is needed when $d=2$. Here we introduce a lower momentum cut-off $1 /(\Lambda L)$ in the integrals (42), (43), with $L$ being a measure of the lateral dimension of the two-dimensional substrate. Proceeding in this way, it can be seen that the integrals (41) now vanish as a power law of $1 / L$. In this regularization scheme, the RG iteration has to be stopped once $\mathrm{e}^{l}=L$. Another possible procedure like that of dimensional regularization can be employed with the same conclusions as those that follow, similar to the RG analysis of tensionless membranes 44 .

In any case, the regularized integrals $\tilde{B}^{(n)}(x, x / 2)$ are no longer identically zero. This leads us to expect the RG flow to escape from the $x=2 K$ plane, which would mean a finite surface tension has been generated under renormalization, and thus the occurence of temperature dependent behavior. Nevertheless, the flow may take many iterations before it appreciably deviates from the $x=2 K$ plane, given that, in its neighborhood, the integrals $\tilde{B}^{(n)}$ can be rather small numbers for large $L$ values.

In Fig. 3 we show the numerical integration of the RG flow (38)-(40) for various initial conditions on the xMBE plane and $L=128$. Indeed, three different types of behavior can be distinguished. For high enough values of $T$, the variables $x, y, K$ are very small and the RG flow escapes from the $x=2 K$ plane very slowly. Under these conditions, the system flows towards the origin while featuring the scale invariant behavior of the $l \mathrm{MBE}$ line $\Gamma_{l \mathrm{MBE}}$. An example is trajectory (1) on Fig. 3. For intermediate temperatures, $\nu \sim x-2 K$ becomes non-negligible and the flow is attracted by the $(x, 0,0)$ segment with $x<1$, where the behavior is described by the EW equation, see trajectory (2) on the figure. Finally, for high values of $T$, the flow falls rapidly onto the $(x, y)$ plane with $x>1$, see trajectory (3) on the figure. This behavior is described by the low temperature massive phase of the sG model, where the lattice potential is dominant and the surface is flat, see Appendix B.

In order to examine this behavior more closely, we can focus on the crossover length $L_{\times}$ introduced in Sec. IIIA. By coarse-graining the system with the RG transformation for scales up to the system size $\left(l^{*}=\ln L\right)$, and if the lattice potential turns out to become irrelevant, we can decide whether the system scaling is of the $l \mathrm{MBE}$ or EW type by evaluating $L_{\times}\left(l^{*}\right)$ to be larger or smaller than $L\left(l^{*}\right) \equiv 1$, respectively. Note that, for a zero lattice potential, the RG flow of $L_{\times}$is easily computed from (29)-(30) to be given by $L_{\times}(l)=L_{\times}(0) \exp (-l)$, hence an exponential behavior of $L_{\times}(l)$ will be a sign of the irrelevance of the lattice potential at scale $l$. In Fig. 1 we show the RG flow of $L_{\times}(l)$ for the same temperature conditions as in Fig. 3 and $L(l=0)=128$. As we see, for high enough temperatures [lines (1), (2) in the figure], indeed $y(l)$ is seen to decay very rapidly to zero, $L_{\times}(l)$ displaying an exponential behavior. However, while for the highest temperature condition [line (1)] the scaling behavior is of the $l \mathrm{MBE}$ type since $L_{\times}\left(l^{*}\right)>1$, we observe that there are lower temperatures for which the lattice potential is irrelevant, but the long distance behavior is rather of the EW type, 
since $L_{\times}\left(l^{*}\right)<1$. This is the case of e.g. the RG flow line (2) on Figs. 3 and 4 , for which the system falls onto the high temperature line of fixed points of the sG model. Finally, for low enough temperatures the lattice potential dominates the asymptotic properties of the system, as signalled in Fig. 田by the complex non-exponential behavior of $L_{\times}(l)$ for $T=0.45$. These temperature condition corresponds in Fig. \& to the RG flow of line (3), along which the system falls onto the flat low temperature phase of the $\mathrm{sG}$ model. In analogy with the sG case, the dynamic RG flow (38)-(40) thus predicts a roughening transition at a temperature

$$
T_{R}=\frac{2 a_{\perp}^{2}\left(\nu+\kappa \Lambda^{2}\right)}{\pi},
$$

at which the flow of the lattice potential changes stability. Note both $\nu$ and $\kappa$ in (44) are renormalized, rather than bare values. For $T<T_{R}$, the system is in the sG massive low temperature phase, within which the surface is flat. For $T \geq T_{R}$, the surface is rough and should asymptotically feature the EW scale invariant behavior. However, crossover behavior exists; specifically, for a small system size (for which the asymptotic behavior may not be reached) or for very high temperatures [for which the RG flow needs many iterations in order to escape the xMBE plane of initial conditions, see line (1) in Fig. 3], the scaling behavior observed in the system will be of the lMBE type.

\section{2. $d>2$ cases}

We have also considered the RG flow of the xMBE model for higher substrate dimensions, finding a behavior which is qualitatively consistent with all the above analysis. Moreover, given that for any $d>2$ the integral $\tilde{\phi}$ in (43) is always a finite number, the results that follow can be taken as additional support for those presented in $d=2$ employing lattice cut-off regularization. As can be seen in Fig. 5, for $d>2$ the RG flow of the xMBE model does take on the exact shape of the generalized model studied in Sec. IIIA, and all the conclusions drawn there become applicable also for the specific initial condition we are now considering. Namely, the RG flow escapes from the $x=2 K$ plane, and thereon the behavior is similar to that of the $\mathrm{sG}$ model. In particular, there is no phase transition and the surface morphology is flat for any value $d>2$. Therefore, the upper critical dimension of the xMBE model (3)-(4) is also predicted to be $d_{c}=2$. Specifically, in Fig. 5, we plot results of a numerical integration of (38)-(40) starting from the xMBE model initial condition for substrate dimensions $d=3$ [panel (a)] and $d=4$ [panel (b)]. As can be seen in the figure, for any initial condition on the xMBE plane - that is, for any temperature value - , the RG flow is towards the sG plane and towards increasing $y$ values, which is the behavior that corresponds to the low temperature flat phase, as in the $\mathrm{sG}$ model for the corresponding substrate dimension. For a given value of $d$, the higher the temperature is, the longer will it take for the RG flow to escape from the plane of initial conditions, since the crossover length $L_{\times}$is larger. In this very high temperature conditions, the initial $l \mathrm{MBE}$ behavior will be relevant for a longer time in the dynamics of Eq. (3).

\section{SUMMARY AND CONCLUSIONS}

The dynamic RG study presented for the generalized model of crystalline surfaces (6)(17) predicts an upper critical dimension $d_{c}=2$. Thus, for a two-dimensional surface there 
exists a roughening temperature, in such a way that the high temperature phase exhibits the scaling properties of the EW equation. Moreover, the transition properties are controlled by the sG fixed point (see Appendix B), and is thus expected to be of a continuous type. If we think of (6)-(7) as a generalization of the $\mathrm{sG}$ model in which a (possibly small) surface diffusion term has been allowed for, we have seen in Sec. IIIA that the consequence is an increase in the $\mathrm{sG}$ roughening temperature, due to the crossover induced by such an irrelevant perturbation. Moreover, the specific case of the xMBE model has been seen to share all these properties, its peculiarity of having a zero bare surface tension merely introducing much more severe crossover effects. From the point of view of applications to epitaxial growth systems, this result illustrates the relevance of EW scaling as an universality class in MBE: in principle, if the symmetry of the system prohibits non-equilibrium surface currents and includes invariance of the dynamics under arbitrary surface tilts, $l \mathrm{MBE}$ scaling is expected [2]. However, we have obtained for a system relaxing linearly as in the $l \mathrm{MBE}$ equation that, if a lattice potential influences the dynamics as in (3) - thereby accounting e.g. for the discrete character of deposition events or the influence of an underlying lattice-, then asymptotic EW scaling should occur. Admittedly, crossovers associated with $l \mathrm{MBE}$ behavior may be nonetheless rather long, particularly for high temperatures.

For the case of the xMBE model, the existence of a phase transition between a flat low temperature phase and a rough high temperature phase as predicted by (38)-(40) is compatible with previous results obtained by the variational approximation 24] and numerical simulations [15, 25]. However, while the variational mean field predicts the transition to be first order, the incorporation of fluctuations by means of the $\mathrm{RG}$ is consistent with a phase transition of a continuous type, which is closer to the numerical results. Moreover, our present RG study predicts the upper critical dimension to be that of the sG model, $d_{c}=2$, while mean-field predicts $d_{c}=4$. We note that the absence of non-trivial mode coupling in the variational mean-field approach limits its capabilities in determining correctly both lower and upper critical dimensions, see e.g. [30] and [24] for the case of the sG model. Concerning numerical simulations [15, 25], EW scaling has been obtained in the high temperature phase only for temperatures which are extremely close to the roughening temperature, whereas $l \mathrm{MBE}$ behavior has been obtained for all other temperature values above the transition. Recall we obtained in Sec. IIIB 1 that, if the temperature was high enough, the system might need a long time to overcome the crossover associated with $l \mathrm{MBE}$ behavior. Seemingly, the finite system sizes thus far employed in the simulations $(L \lesssim 128)$ are affected by this type of crossover limitations. We also note that the occurrence of EW scaling for the xMBE model is reminiscent of the hexatic phase claimed for the Lr model [20, 21, 22]. This is an intermediate phase which features EW scaling, and which lies in between the flat low temperature phase (the liquid phase in the melting context) and the high temperature phase (solid phase) with $l \mathrm{MBE}$ scaling, being separated from both of them by roughening transitions of the KT type [20, 21, 22]. On the other hand, as found in 455 and references therein, there are also claims on the inexistence of an hexatic phase in the Lr model and, moreover, on the first order type of the only roughening transition ensuing. We are currently performing large scale simulations of the Lr model on the square lattice 29] in order to check the predictions of our RG analysis, in particular whether any trace of crossover behavior and EW scaling can be detected in the surface properties at high temperatures. 


\section{Acknowledgments}

We are grateful to Juan Jesús Ruiz-Lorenzo and Angel Sánchez for discussions and encouragement, and to Martin Rost for discussions and comments. E.M. aknowledges the EU fellowship No. HPMF-CT-2000-0487. This research has been supported by EPSRC (UK) grant No. GR/M04426, DGES (Spain) grant No. HB1999-0018, and by MCyT (Spain) grant No. BFM2000-0006.

\section{APPENDIX A}

In this Appendix we recall the considerations needed to perform the correct Taylor expansions in (19), using Kadanoff's operator algebra [32]. We generalize results for the static RG analysis of the sine-Gordon nonlinearity 8, 33. Thus, in our dynamical RG calculation each of the (infinite number of) non-linearities $O_{2 n+1}(\boldsymbol{\rho})=\left(\bar{h}-\bar{h}^{\prime}\right)^{2 n+1}$ appearing in the Taylor expansion of $\sin \left[2 \pi\left(\bar{h}-\bar{h}^{\prime}\right) / a_{\perp}\right]$ contributes a term proportional to the marginal operator $O_{1}(\boldsymbol{\rho})=\bar{h}-\bar{h}^{\prime}$, where, as above, $\boldsymbol{\rho}=\mathbf{r}-\mathbf{r}^{\prime}$. In our continuum approach, this is the most relevant term originating (via Taylor expansion) the renormalization of both the $\Delta h$ and $\Delta^{2} h$ terms in Eq. (6). Thus,

$$
\begin{aligned}
O_{2 n+1}(\boldsymbol{\rho}) & \equiv\left(\bar{h}(\mathbf{r})-\bar{h}\left(\mathbf{r}^{\prime}\right)\right)^{2 n+1} \\
& =\tilde{O}_{2 n+1}(\mathbf{r})+a_{2 n+1}(\boldsymbol{\rho})\left(\bar{h}-\bar{h}^{\prime}\right),
\end{aligned}
$$

where $\tilde{O}_{2 n+1}(\mathbf{r})$ is an irrelevant operator. A way to compute the $a_{2 n+1}$ constants is 32, 33 by performing all contractions contributing to the behavior

$$
\left\langle O_{2 n+1}(\mathbf{r}) O_{1}(\mathbf{r}+\boldsymbol{\rho})\right\rangle_{\bar{h}} \sim a_{2 n+1}(\boldsymbol{\rho})\left\langle O_{1}(\mathbf{r}) O_{1}(\mathbf{r}+\boldsymbol{\rho})\right\rangle_{\bar{h}}
$$

where, within our order of approximation in powers of $V$, averages $\langle\cdots\rangle_{\bar{h}}$ are computed with respect to the Gaussian distribution of $\bar{h}$ given by the $V=0$ limit of (7)). Thus one obtains

$$
a_{2 n+1}(\boldsymbol{\rho})=(2 n+1)\left\langle\left(\bar{h}-\bar{h}^{\prime}\right)^{2 n}\right\rangle_{\bar{h}}=\frac{(2 n+1) !}{2^{n} n !}\left\langle\left(\bar{h}-\bar{h}^{\prime}\right)^{2}\right\rangle_{\bar{h}}^{n}
$$

Using this result in the Taylor expansion of the sine, one gets

$$
\begin{aligned}
\sin \left[\frac{2 \pi\left(\bar{h}-\bar{h}^{\prime}\right)}{a_{\perp}}\right] & =\sum_{n=0}^{\infty} \frac{(-1)^{n}}{(2 n+1) !}\left(\frac{2 \pi}{a_{\perp}}\right)^{2 n+1} O_{2 n+1}(\boldsymbol{\rho}) \\
& \sim \sum_{n=0}^{\infty} \frac{(-1)^{n}}{2^{n} n !}\left(\frac{2 \pi}{a_{\perp}}\right)^{2 n+1}\left\langle\left(\bar{h}-\bar{h}^{\prime}\right)^{2}\right\rangle_{\bar{h}}^{n} O_{1}(\mathbf{r}) \\
& =\frac{2 \pi}{a_{\perp}} O_{1}(\mathbf{r}) \exp \left[-\frac{2 \pi^{2}}{a_{\perp}^{2}}\left\langle\left(\bar{h}-\bar{h}^{\prime}\right)^{2}\right\rangle_{\bar{h}}\right]
\end{aligned}
$$

This is the result employed in Eq. (20) of Sec. 11 . 


\section{APPENDIX B}

As already stated, the dynamic RG flow (38)-(40) can be seen as a generalization of that for the $\mathrm{sG}$ equation, as derived e.g. in [8, 9]. In this Appendix we explore this relationship in some more detail in the physically interesting case $d=2$.

Similarly to the sG case, an important point in the $(x, y, K)$ parameter space is that where the lattice potential $y$ changes stability. Actually, the existence in the generalized model of a surface diffusion term does not change this fact, in the sense that the point $(1,0,0)$ still controls the behavior of the RG flow to a large extent. This is due to the facts that the surface diffusion is irrelevant with respect to surface tension, and that the latter is generated by the RG flow, as obtained in Sect. [II. The simplest way to substantiate this conclusion is to study the flow (38)-(40) perturbatively near the point $(1,0,0)$. To this end, we introduce the temperature-like variable [8, 9] $t \equiv 2 / x-2$, rewrite Eqs. (38)-(40) in the new variables $(t, y, K)$, and approximate the corresponding flow to second order around the fixed point $(t, y, K)=(0,0,0)$. We obtain

$$
\begin{aligned}
\frac{\mathrm{d} t}{\mathrm{~d} l} & =8 K(1+t)-y^{2}\left(B^{(2)}-\frac{B^{(4)}}{16}\right), \\
\frac{\mathrm{d} y}{\mathrm{~d} l} & =-t y \\
\frac{\mathrm{d} K}{\mathrm{~d} l} & =-2 K-\frac{y^{2}}{64} B^{(4)}
\end{aligned}
$$

where we have termed $B^{(n)} \equiv \tilde{B}^{(n)}(1,0)$. Introducing new variables in order to bring flow (B1)-(B3) into normal form [41]

$$
\begin{aligned}
t & =u_{1}+\frac{1}{2} u_{1}^{2}, \\
y & =u_{2}, \\
K & =u_{3}-\frac{B^{(4)}}{128} u_{2}^{2},
\end{aligned}
$$

we finally obtain

$$
\begin{aligned}
& \frac{\mathrm{d} u_{1}}{\mathrm{~d} l}=8 u_{3}-B^{(2)} u_{2}^{2}, \\
& \frac{\mathrm{d} u_{2}}{\mathrm{~d} l}=-u_{1} u_{2}, \\
& \frac{\mathrm{d} u_{3}}{\mathrm{~d} l}=-2 u_{3} .
\end{aligned}
$$

The interesting feature of the approximate flow (B4)-(B6) is that the third equation can be readily solved for the surface diffusion-like variable $u_{3}$, as $u_{3}(l)=u_{3}(0) \exp (-2 l)$, thus making apparent one of the qualitative features of the original RG flow, namely the fact that surface diffusion is an irrelevant variable that decouples (exponentially) fast from the flow for the lattice potential and the surface tension. On the other hand, by taking the ratio between the first and second equations in (B4)-( $(\bar{B} 6)$ and integrating in $l$, one can check that the relation

$$
u_{1}^{2}(l)=B^{(2)} u_{2}^{2}(l)-16 \int_{l}^{\infty} u_{1}\left(l^{\prime}\right) u_{3}\left(l^{\prime}\right) \mathrm{d} l^{\prime}
$$


defines a separatrix for the flow (B4)-(B6), generalizing the well-known asymptotes of the sG hyperbolae [9] [see also panel (a) of our Fig. 1]. On e.g. the first quadrant of the $\left(u_{1}, u_{2}\right)$ plane, flow lines below the separatrix flow onto the $u_{2}=0$ line of fixed points (irrelevant lattice potential, high temperature behavior), whereas flow lines above the separatrix flow towards large values of the lattice potential $u_{2}$ (low temperature, massive phase). Thus, the separatrix marks the temperature driven phase transition. Unfortunately, we have not been able to produce a useful simpler expression for locus (B7), not even within perturbation theory.

[1] A.-L. Barabási and H.E. Stanley, Fractal concepts in surface growth (Cambridge University Press, Cambridge, 1995).

[2] J. Krug, Adv. Phys. 46, 139 (1997).

[3] J. García-Ojalvo and J.M. Sancho, Noise in Spatially Extended Systems (Springer, New York, 1999).

[4] J. Marro and R. Dickman, Nonequilibrium phase transitions in lattice models (Cambridge University Press, Cambridge, 1999).

[5] J.D. Weeks, in Ordering in strongly fluctuating condensed matter systems, edited by T. Rice (Plenum, New York, 1980).

[6] G. Grinstein and D.H. Lee, Phys. Rev. Lett. 66, 177 (1991).

[7] S.T. Chui and J.D. Weeks, Phys. Rev. Lett. 40, 733 (1978).

[8] P. Nozières and F. Gallet, J. Physique (Paris) 48, 353 (1987).

[9] P. Nozières, in Solids far from equilibrium, edited by C. Godrèche (Cambridge University Press, Cambridge, 1991).

[10] A. Sánchez, A.R. Bishop, and E. Moro, Phys. Rev. E 62, 3219 (2000).

[11] S.F. Edwards and D.R. Wilkinson, Proc. R. Soc. London A 381, 17 (1982).

[12] See K. Honda, Phys. Rev. E 55, R1235 (1997) for a precise interpretation of the negative roughness exponents expected for $d>2$ in the case of the EW equation.

[13] Y. Saito, Statistical Physics of Crystal Growth (World Scientific, Singapore, 1996).

[14] A. Pimpinelli and J. Villain, Physics of crystal growth (Cambridge University Press, Cambridge, 1998).

[15] E. Moro, R. Cuerno, and A. Sánchez, Phys. Rev. Lett. 78, 4982 (1997).

[16] W.W. Mullins, J. Appl. Phys. 28, 333 (1957).

[17] Reflection High-Energy Electron Diffraction and Reflection Electron Imaging of Surfaces, edited by P.K. Larsen and P.J. Dobson, NATO ASI Ser. B Vol. 188 (Plenum, New York, 1988).

[18] L.-H. Tang and T. Nattermann, Phys. Rev. Lett. 66, 2899 (1991).

[19] H. Kallabis, L. Brendel, J. Krug, and D.E. Wolf, Int. J. Mod. Phys. B 11, 3621 (1997).

[20] D. Nelson, Phys. Rev. B 26, 269 (1982).

[21] D.A. Bruce, Mater. Sci. Forum 4, 51 (1985).

[22] K.J. Strandbrug, Rev. Mod. Phys. 60, 161 (1988).

[23] H. Kleinert, Gauge fields in condensed matter (World Scientific, Singapore, 1989).

[24] E. Moro and R. Cuerno, Phys. Rev. E 63, 036104 (2001).

[25] E. Moro, Ph.D. thesis, Universidad Carlos III de Madrid, 1999 (unpublished, in Spanish); http://www-thphys.physics.ox.ac.uk/users/EstebanMoro/profesional.html 
[26] J. Villain, J. Phys. (Paris) I 1, 19 (1991).

[27] Z.-W. Lai and S. Das Sarma, Phys. Rev. Lett. 66, 2348 (1991).

[28] S. Majaniemi, T. Ala-Nissila, and J. Krug, Phys. Rev. B 53, 8071 (1996).

[29] E. Moro, J.J. Ruiz-Lorenzo, R. Cuerno, and A. Sánchez (to be published).

[30] Y. Saito, Z. Phys. B: Condens. Matter 32, 75 (1978).

[31] L. Vázquez, R.C. Salvarezza, and A.J. Arvia, Phys. Rev. Lett. 79, 709 (1997).

[32] L.P. Kadanoff, Statistical Physics: Statics, Dynamics and Renormalization (World Scientific, Singapore, 2000).

[33] H.J.F. Knops and L.W.J. den Ouden, Physica A, 103597 (1980).

[34] Y. Levin and K.A. Dawson, Phys. Rev. A 42, 3507 (1990).

[35] B. Kahng and K. Park, Int. J. Mod. Phys. B 10, 543 (1996).

[36] T. Sun, B. Morin, H. Guo, and M. Grant, in Surface disordering: growth, roughening and phase transitions, edited by R. Jullien et al. (Nova Science, New York, 1992).

[37] M. Rost and J. Krug, J. Physique I 7, 1627 (1997).

[38] J.M. Kim and S. Das Sarma, Phys. Rev. E 48, 2599 (1993).

[39] A.Kr. Chattopadhyay, cond-mat/0001020.

[40] D.E. Wolf and J. Villain, Europhys. Lett. 13, 389 (1990).

[41] A.H. Nayfeh, Method of normal forms (John Wiley \& Sons, New York, 1993).

[42] R.H. Swendsen, Phys. Rev. B 18, 492 (1987).

[43] H. Müller-Krumbhaar, in 1976 Crystal Growth and Materials, edited by E. Kaldis and H.J. Scheel (North-Holland, New York, 1977).

[44] S. Leibler, in Statistical mechanics of membranes and surfaces, edited by D. Nelson, T. Piran, and S. Weinberg (World Scientific, Singapore, 1989).

[45] W. Janke and H. Kleinert, Phys. Rev. B 41, 6848 (1990). 

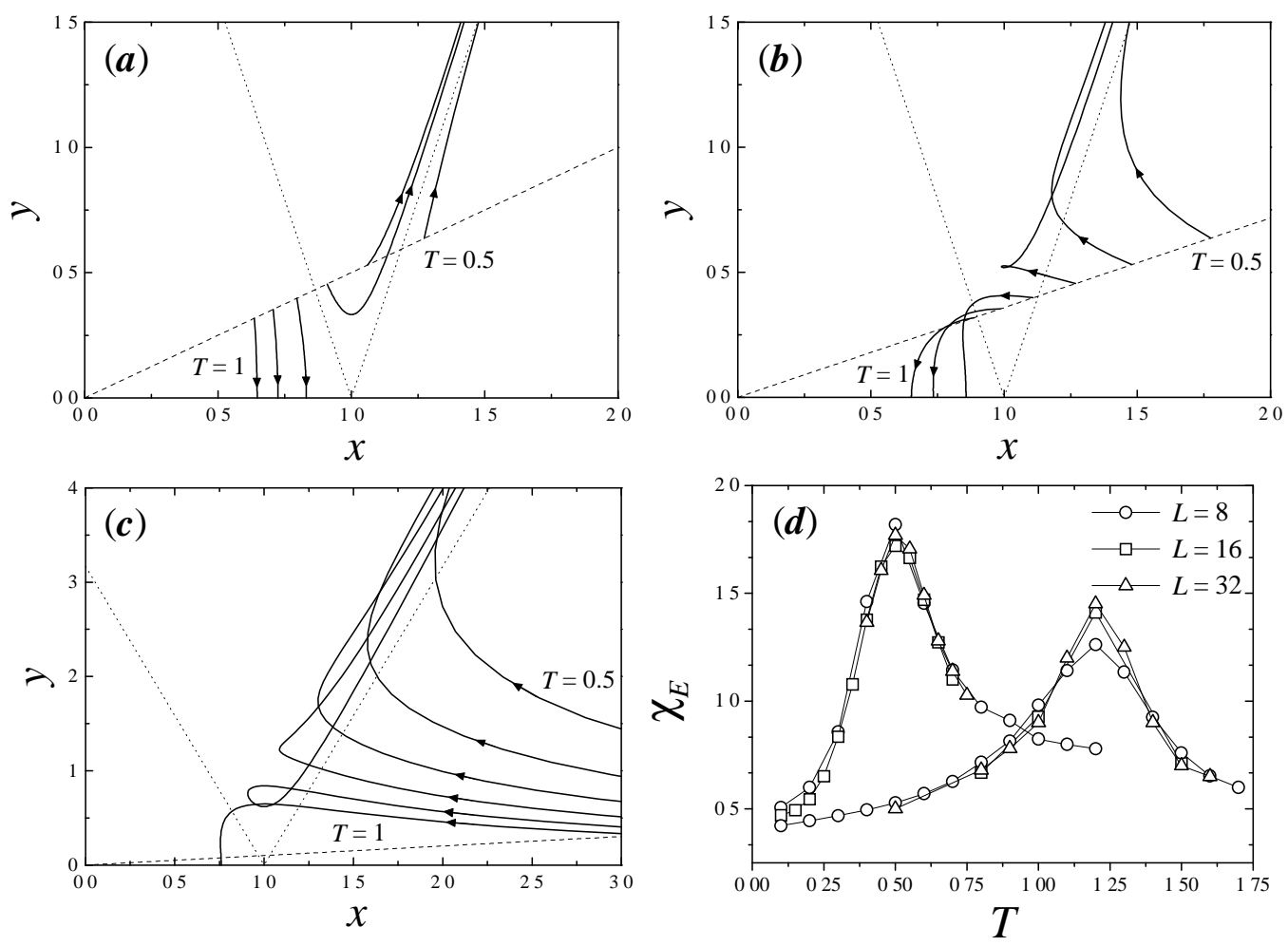

FIG. 1: Panels (a)-(c): $(x, y)$ projection of the RG flow (38)-(40) for $d=2$, using $\nu(0)=V(0)=$ $1=a_{\perp}=\Lambda=1$, and (a) $\kappa(0)=0$, (b) $\kappa(0)=10^{-2}$, (c) $\kappa(0)=10^{-1}$. In panels (a)-(c), initial conditions lie on the dashed line and the dotted line is the separatrix for the pure sG flow. Solid lines correspond to $T=0.5,0.6,0.7,0.8,0.9$, and $T=1$ right to left in (a) and (b), and top to bottom in (c). Panel (d): Specific heat for the sG model (left curves) and for model (66) with $\mu=\nu=1$ and $\kappa=0.5$ (right curves) for different system sizes. All units employed are arbitrary. 

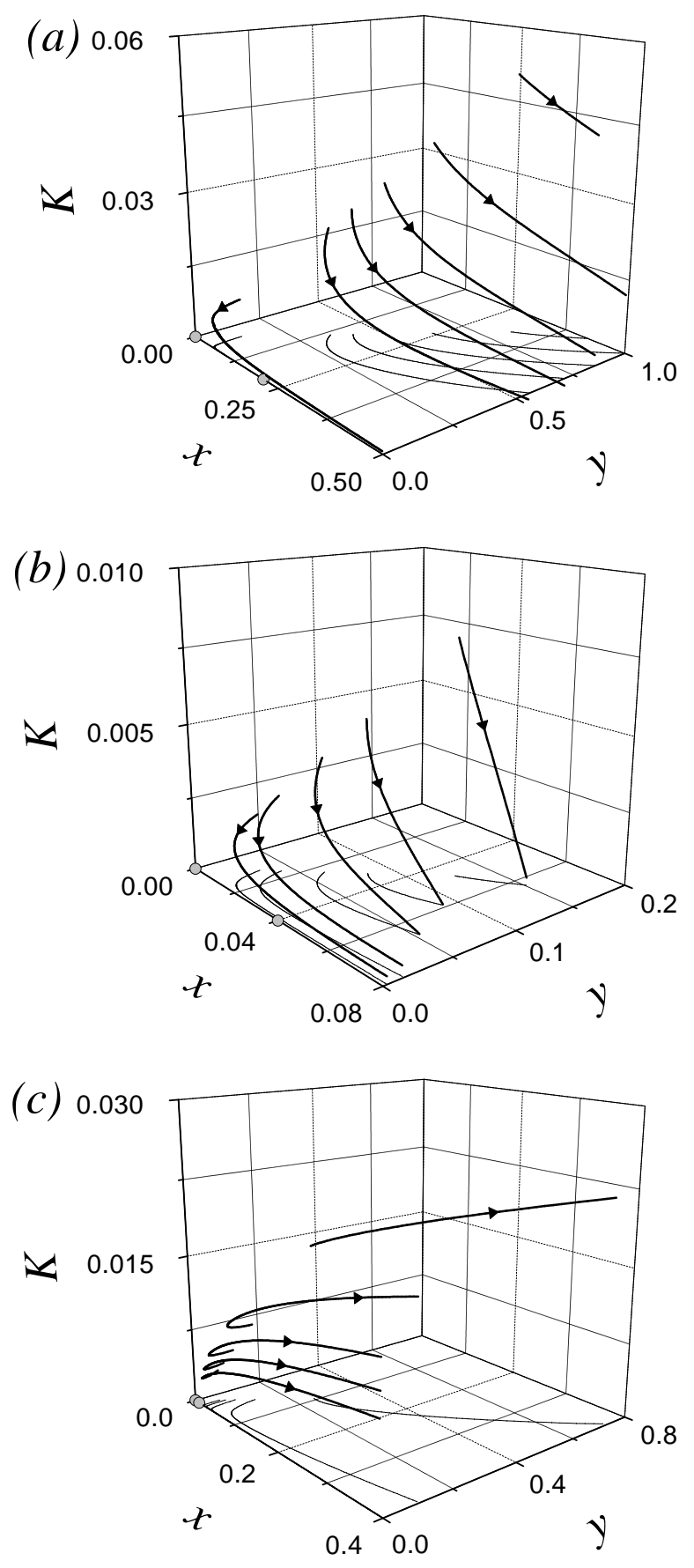

FIG. 2: RG trajectories from a numerical integration of (38)-(40) for $d=3$ (a), $d=4$ (b), and $d=5$ (c) with $\nu(0)=0.5, \kappa(0)=0.25$, and $V(0)=0.1$. Thick solid lines correspond, right to left, to (a) $T=1.5,2,2.5,3,3.5,10$; (b) $T=10,15,20,30,40$; (c) $T=5,10,15,20$, 25. Thin solid lines on the $(x, y)$ planes are projections of the RG flow lines above them. Thick dots on the $x$ axis denote both the origin and the point $x=2 \mathcal{S}_{d} \Lambda^{d-2} /\left[d(2 \pi)^{d-1}\right]$ at which the rhs of the flow equation for $y$ vanishes. Other parameters are as in Fig. 1. All units employed are arbitrary. 


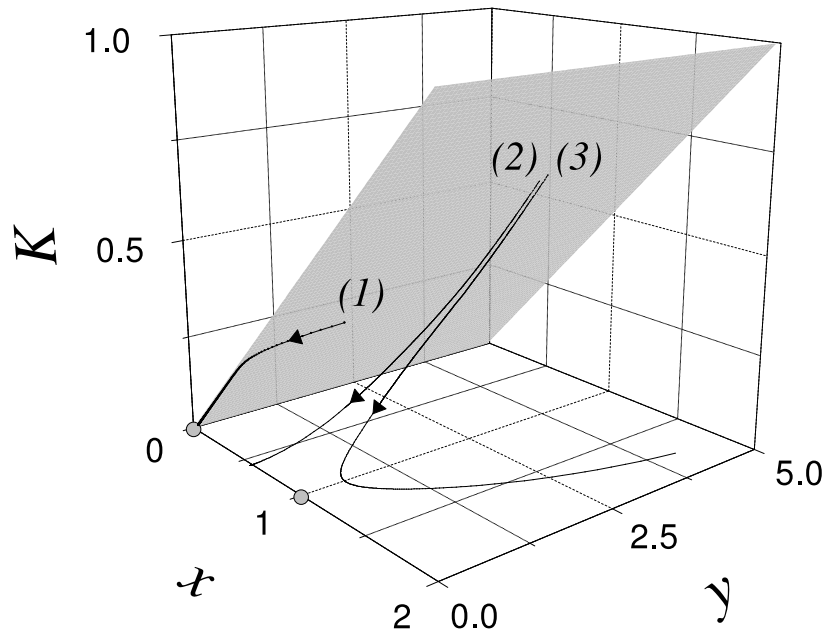

FIG. 3: RG trajectories from a numerical integration of (38)-(40) for the xMBE model [i.e. $\nu(0)=0$ ] with $L=128, \kappa(0)=1$, and $V(0)=0.1$. Solid lines exemplify the three types of behavior depending on $T$ : (1) high $T$ phase, $l \mathrm{MBE}$ scale invariant behavior $(T=10)$; $(2)$ intermediate $T$ phase, EW scale invariant behavior $(T=0.46)$; $(3)$ low $T$ massive phase $(T=0.45)$. For the sake of clarity, all three coordinates along line (1) have been artificially expanded by a factor of 10 . The $x=2 K$ plane of initial conditions appears shaded, and both the origin and the $(1,0,0)$ point are signalled with thick dots. Other parameters are as in Fig. 1. All units employed are arbitrary.

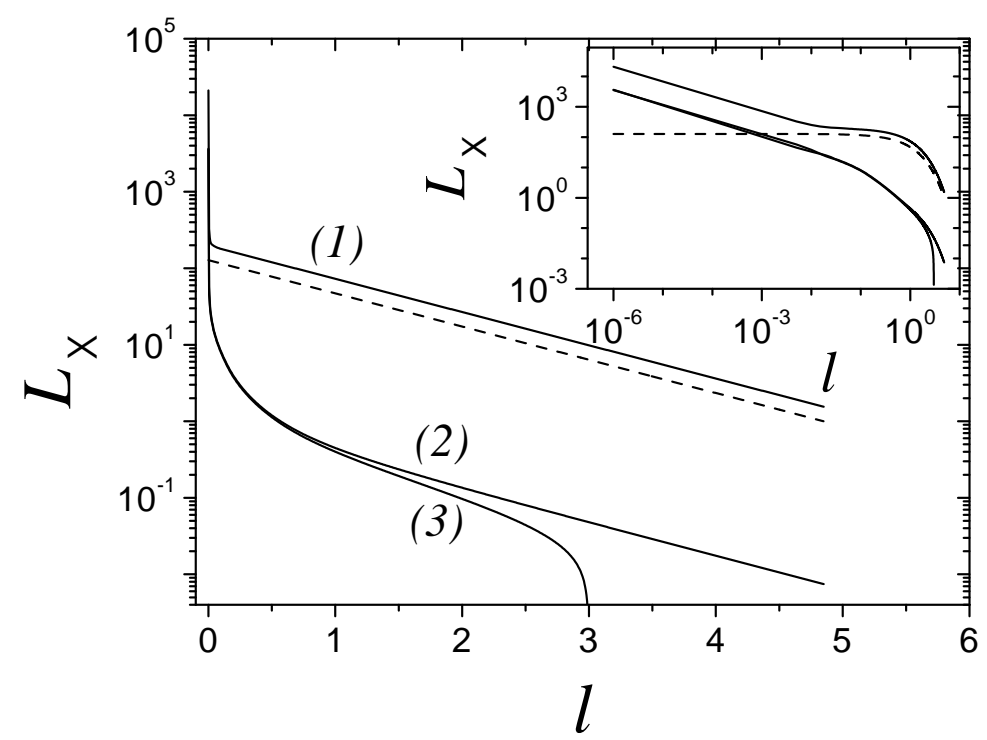

FIG. 4: Linear-log plot of the numerical RG flow of $L_{\times}(l)$ for $d=2, L=128$. Other parameters are as in Fig. 3. Solid lines are for $T=10,0.46$, and $T=0.45$, top to bottom. For the sake of comparison, the dashed line depicts the RG flow of $L_{\times}(l)$ for the linear system $V(0)=0$. In all cases the flow is terminated for $l=\ln 128 \simeq 4.85$. All units employed are arbitrary. Inset: Blow-up of the same plot for small $l$ values, in log-log representation. 

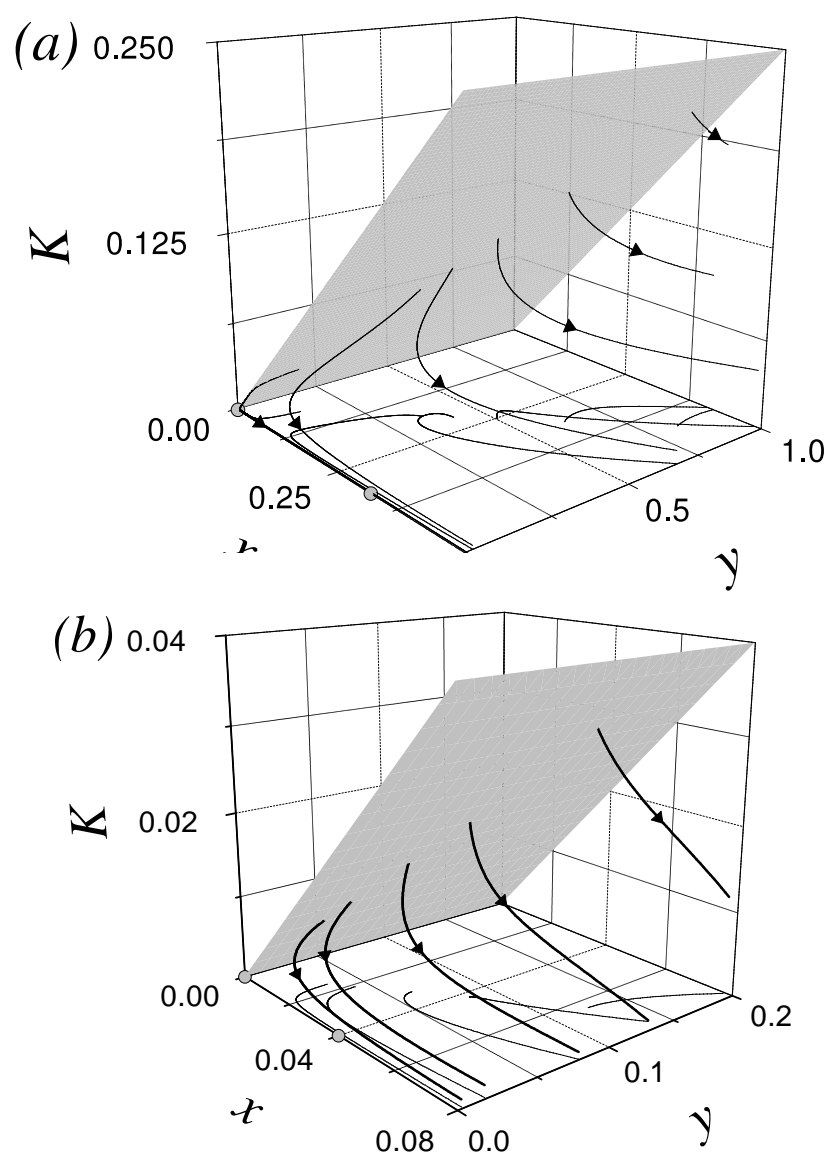

FIG. 5: RG trajectories from a numerical integration of (38)-(40) for the xMBE model [i.e. $\nu(0)=0$ ] with $\kappa(0)=1$, and $V(0)=0.1$. Panel (a) [(b)] corresponds to $d=3[d=4]$. On each panel, solid lines correspond to temperature values $T=1.5,2,2.5,3,3.5$, and $T=10$, right to left. Thin solid lines on the $(x, y)$ plane are projections of the RG flow lines above them. As in Fig. 3, the $x(0)=2 K(0)$ plane of initial conditions appears shaded. Thick dots on the $x$ axis denote both the origin and the point $x=2 \mathcal{S}_{d} \Lambda^{d-2} /\left[d(2 \pi)^{d-1}\right]$ at which the rhs of the flow equation for $y$ vanishes. Other parameters are as in Fig. 1. All units employed are arbitrary. 